\title{
OPTIMAL AND ROBUST DAMPING CONTROL FOR SEMI-ACTIVE VEHICLE SUSPENSION
}

\author{
Uwe Rettig ${ }^{\dagger}$ \\ Axxom Software AG \\ München, Germany \\ Uwe.Rettig@axxom.com \\ $\dagger$ research performed during affiliation with TU München
}

\author{
Oskar von Stryk* \\ Department of Computer Science \\ Technische Universität Darmstadt \\ Germany, * contact author \\ stryk@sim.tu-darmstadt.de
}

\begin{abstract}
The paper focusses on optimal control issues arising in semi-active vehicle suspension motivated by the application of continuously controllable ERF-shock absorbers. Optimality of the damping control is measured by an objective consisting of a weighted sum of criteria related to safety and comfort which depend on the state variables of the vehicle dynamics model. In the case of linear objectives and linear quarter or half car dynamics models the well-known linear quadratic regulators can be computed. However, to account for maximum robustness with respect to unknown perturbations, e.g., by the ground, linear robust-optimal $\mathrm{H}$-infinity controllers are investigated which can be computed iteratively. The linear H-infinity controller can be viewed as the solution of a linear dynamic zero-sum differential game. Thus, a nonlinear $\mathrm{H}$-infinity controller can be obtained in principle as the solution of a nonlinear zero-sum dynamic game problem. Such a problem formulation enables to consider nonlinear vehicle dynamics as well as nonlinear objectives and constraints. A computational method is discussed which computes approximations of robust-optimal trajectories for nonlinear damping control. The method is based on a reformulation of the dynamic game and the application of a control and state parameterization approach in combination with sparse nonlinear programming methods. Numerical results for the different approaches and their validation by software-in-the-loop simulation using a full motor vehicle dynamics model are presented.
\end{abstract}

\section{Key words}

semi-active suspension, ERF shock absorber, vehicle dynamics, optimal control, robust-optimal control, direct transcription

\section{Introduction}

Main improvements in vehicle comfort and safety can be obtained by electronic control units which continuously or discretely in time interfere with the vehicle dy-

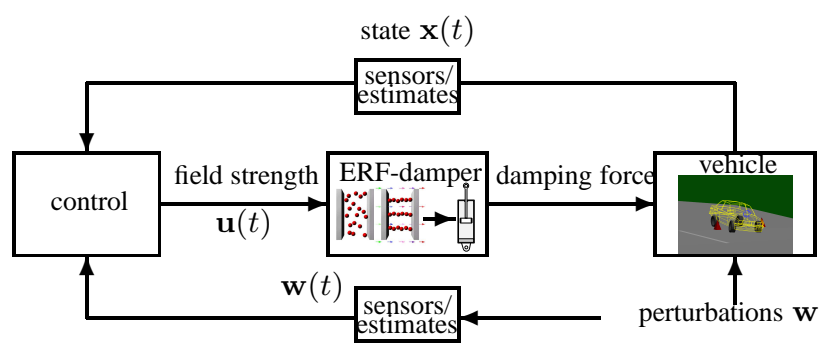

Figure 1. Control, perturbation, and state variables for the semiactive vehicle suspension system using ERF shock absorbers.

namics. Damping of vibrations in active or semi-active suspensions is such a subject for research in control and highly relevant for vehicle comfort and safety.

Conventional, passive shock absorbers exhibit a given damping characteristic, i.e., the damping force as a given function of the piston rod velocity. Thus, they can only represent a compromise between different objectives as comfort, safety or speed. A significant improvement of the vehicle's suspension can be obtained by adding active components as hydraulic or electromagnetic actuators. However, generation of the additional forces requires an additional energy supply. Using electronically controlled active dampers the damping characteristic can be adapted to the actual dynamic state of the vehicle to improve ride comfort or safety. Here, we consider the protoype of a continuously controllable shock absorber on the basis of electrorheological fluids (ERF). The viscosity of the synthetic ERF can be changed by an applied electrical field within milliseconds. By controlling an electrical field applied to one or several valves of a shock absorber equipped with an ERF the damping characteristic can be adapted within milliseconds to the current ride state. Unlike hydraulic systems the ERF shock absorber does not require significant additional space for installation. As it does not add energy to the overall system such a suspension is semi-active. However, for investigating suitable controls a dynamic model of the ERF shock absorber must be developed which describes the relation between applied electric field strength, damper posi- 
tion and velocity and the resulting damping force (Sect. 2.1). One of the most common control concepts for active and semi-active vehicle shock absorbers is the socalled skyhook damping control (e.g., [Valasek et al., 1997]), which controls heuristically the movements of the vehicle body. Analogously the groundhook control concept takes into account wheel oscillations (e.g., [Valasek et al., 1997]). Investigations by [Alleyne and Hedrick, 1995] consider a nonlinear adaptive control scheme, due to unknown observer-based parameters. The concept of linear quadratic optimal regulators for active damping control is common in scientific literature, e.g., in [Hać, 1992] in the context of active preview control.

To evaluate different strategies for active or semiactive control, mathematical models of objectives for ride comfort and safety are required (Sect. 2.3). But ride safety (e.g. provided by large contact forces between tire and road) and ride comfort (e.g., provided by small vertical accelerations of the vehicle body) are mainly antagonistic. Furthermore, the design of optimal active suspension controls must consider the relevant properties of the vehicle dynamics (Sect. 2.2) as well as the usually unknown disturbances from an uneven road. As the road properties cannot be predicted accurately and economically enough by onboard sensors robustness of the active damping control with respect to unknown disturbances is important to guarantee a certain performance of the ride.

\section{Optimal Control Problems for Optimal Semi- Active Suspension}

The investigation of optimal semi-active suspension on the basis of ERF shock absorbers requires to consider the dynamic behavior of the vehicle as well as of the ERF shock absorber (Fig. 1).

\subsection{Dynamic Model of a Continuously Control- lable ERF Shock Absorber}
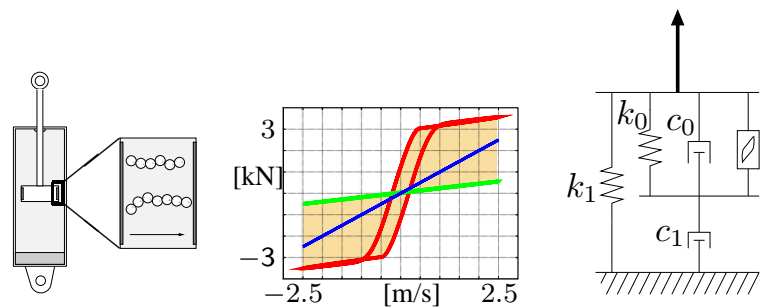

Figure 2. The variable viscosity of the ERF flow through a valve between two electrodes (left) enables a broad bandwidth of forcevelocity characteristic of the shock absorber (middle) which can be described by an augmented Bouc-Wen dynamic model (right).

Conventional, passive damping behavior is usually described by a fixed damping characteristic defined by the force-velocity rate. But in general, dampers exhibit a nonlinear behavior. Depending on the actual damper design, the damping rates are different in the bump and rebound phases while within each phase the system is best described by a progressive damping characteristic. For a more realistic simulation model, piecewise linear or higher order polynomial approximations of realistic tabular data may be used.

Measurements of ERF dampers indicate strong nonlinear behavior and characteristic with hysteresis. The dynamics of the characteristic depends upon the damping velocity and on the applied electrical field. Detailed computations of ER fluid flow models within the valve are not well suited for the purpose of control design because of the high computational and analytical efforts involved in deriving proper models and obtaining numerical solutions [Hoppe et al., 2000]. However, a sufficiently accurate and fast computable model of the dynamic behavior of an ERF shock absorber can be obtained, e.g., using parameterized, phenomenological models. Here the approximation of the ERF effects is usually induced by friction elements or nonlinear spring or damper elements [Stanway, Spronston and El-Wahed, 1995; Butz and von Stryk, 2002]. The augmented Bouc-Wen model by [Spencer et al. 1996b] (see also [Butz and von Stryk, 2002; Hoppe et al., 2000] and Fig. 2 (right)) is one of the most flexible models which take into account the dependency on a variable electrical field. It describes a dynamic system depending on $\dot{x}_{\mathrm{D}}$, the velocity of the piston rod. The state variable of the dynamic ERF damper model is denoted by $\mathbf{s}=\left(s_{1}, s_{2}, s_{3}\right)^{\mathrm{T}}$

$$
\left.\begin{array}{l}
\dot{s}_{1}=\frac{1}{c_{0}+c_{1}}\left(c_{1} \dot{x}_{\mathrm{D}}-\alpha s_{2}-k_{0} s_{1}\right) \\
\dot{s}_{2}=\left(A-\beta\left(1+\operatorname{sgn}\left(\dot{s}_{1} s_{2}\right)\right) s_{2}^{2}\right) \dot{s}_{1} .
\end{array}\right\}
$$

The output function

$$
F=c_{1}\left(\dot{x}_{\mathrm{D}}-\dot{s}_{1}\right)+k_{1}\left(x_{\mathrm{D}}-x_{0}\right)
$$

denotes the damping force and depends on $\dot{x}_{\mathrm{D}}$ and $x_{\mathrm{D}}$, the relative displacement of the damper. The system (1) describes a hysteresis operator, and its properties are parameterized with respect to the applied electrical field. Thus

$$
\dot{s}_{3}=\eta\left(u_{\mathrm{D}}-s_{3}\right)
$$

results with control $u_{\mathrm{D}}$ as the applied field strength and

$$
\left.\begin{array}{rl}
c_{0} & =c_{01}+s_{3} c_{02} \\
c_{1} & =c_{11}+s_{3} c_{12} \\
\alpha & =\alpha_{1}+s_{3} \alpha_{2} .
\end{array}\right\}
$$

For modeling the dynamic behavior of a particular ERF damper prototype the 11-dimensional parameter vector $\mathbf{p}=\left(c_{11}, c_{01}, \alpha_{1}, A, \beta, c_{11}, c_{02}, \alpha_{2}, k_{0}, k_{1}, x_{0}, \eta\right)^{\mathrm{T}}$ must be optimized numerically in such a way that the simulated trajectories fit a set of measured damper trajectories best using a nonlinear least squares objective [Rettig, 2003]. 


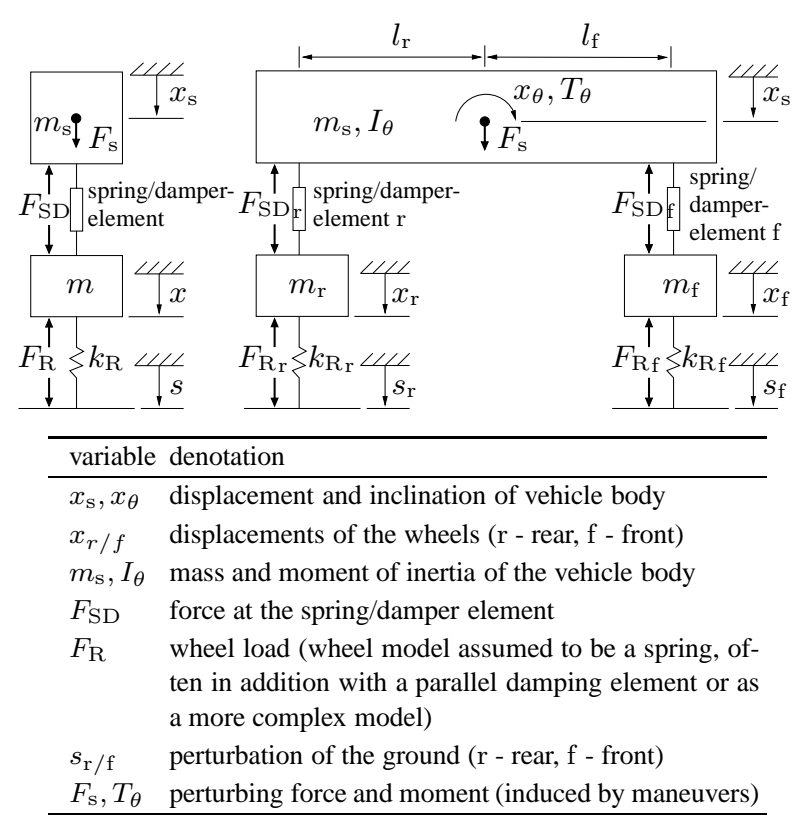

Figure 3. Quarter car model (left) and half car model (right).

\subsection{Models of the Vehicle Dynamics}

Motor vehicles are very complex dynamic systems and consist of many significant components resulting in a high number of degrees of freedom. A detailed and comprehensive vehicle model is needed to represent the nonlinear kinematics of the wheels and axles and to describe the drive train, the steering mechanism, the tire dynamics and ground contact forces [Genta, 1997; Kortüm and Lugner, 1998]. Depending upon the specific design purpose of interest, a suitable dynamical model can be achieved by a reduced dynamic system. The effect of the neglected components of the full model may be interpreted as general perturbations applied to the reduced system.

2.2.1 Models of Quarter Cars and Linear Vehicle Dynamics The oscillating behavior of a vehicle excited by perturbations of the ground and driving maneuvers are investigated using vertical dynamics mod$e l s$, i.e. models reduced to the vertical displacements of the vehicle and wheel bodies. The unknown maneuvers such as braking, acceleration and cornering under high velocities, if considered, may be regarded as general perturbations.

Vertical models of vehicles may be classified into different levels of detail. There are so-called full-carmodels (in the context of vertical models) including two axles, which reflect both vertical deflections and inclinations. Bounce, roll and pitch motions can be investigated simultaneously. Separated and decoupled investigations are possible using half-car-models (cf. Fig. 3, right). The inclination is interpreted as roll or pitch motion. The most common and simple model is the quarter-car-model (cf. Fig. 3, left), which represents the vertical motion of a system including a quarter of the vehicle body and the corresponding wheel. In the computations of subsequent chapters for the wheel the constants $k_{\mathrm{R}}=150 \mathrm{kN} / \mathrm{m}$ (spring), $c_{\mathrm{R}}=0.2 \mathrm{kNs} / \mathrm{m}$ (damping), $m_{\mathrm{R}}=35 \mathrm{~kg}$ (mass) and for the suspension $k_{\mathrm{s}}=20 \mathrm{kN} / \mathrm{m}$ (spring) and $c_{\mathrm{s}}=1.5 \mathrm{kNs} / \mathrm{m}$ (damping constant in case of a passive suspension) and a quarter vehicle mass of $m_{\mathrm{s}}=324 \mathrm{~kg}$ have been used.

2.2.2 Full Motor Vehicle Dynamics Model The numerical, real-time simulation of a full motor vehicle dynamics model that accounts for all significant effects is used in our investigations to validate optimal and suboptimal controls that have been computed using reduced models of the vehicle dynamics. Our vehicle model consists of a suitable multibody system with kinematical connections and force elements and is supplemented by a sophisticated tire model. A general purpose modeling approach to multibody systems based on the descriptor form of the equations of motion results in a large-scale system of differential-algebraic equations (DAEs) of index 3. However, we make use of an optimally tailored model description which yields a system of ordinary differential equations (ODEs) and is well suited for simulation in real-time.

The vehicle model of veDYNA [Chucholowski et al., 1999; Vögel et al., 2003] consists of a system of nine rigid bodies comprising the vehicle body, the axle suspensions and the wheels. Further submodels are employed to depict the characteristic of the drive train, the steering mechanism, and the tires. Suitable minimal coordinates and generalized velocities are used to describe the spatial state of the vehicle and its components [Rill, 1994]. The equations of motion are derived from Jourdain's Principle yielding

$$
\begin{aligned}
\mathbf{M}_{B V}\left(\mathbf{y}_{B V}\right) \dot{\mathbf{z}}_{B V} & =\mathbf{Q}_{B V}\left(\mathbf{y}_{B V}, \mathbf{z}_{B V}, \mathbf{y}_{S T}\right. \\
\left.\mathbf{z}_{S T}, \mathbf{y}_{D T}, \mathbf{z}_{D T}\right) & (5-\mathrm{a}) \\
\dot{\mathbf{y}}_{B V} & =\mathbf{K}_{B V}^{-1}\left(\mathbf{y}_{B V}\right) \mathbf{z}_{B V} \\
\mathbf{M}_{D T} \dot{\mathbf{z}}_{D T} & =\mathbf{Q}_{D T}\left(\mathbf{y}_{D T}, \mathbf{z}_{D T}\right) \\
\dot{\mathbf{y}}_{D T} & =\mathbf{V}_{D T} \mathbf{z}_{D T} \\
\mathbf{M}_{S T}\left(\mathbf{y}_{S T}, \mathbf{y}_{B V}\right) \dot{\mathbf{z}}_{S T} & =\mathbf{Q}_{S T}\left(\mathbf{y}_{S T}, \mathbf{z}_{S T}\right) \\
\dot{\mathbf{y}}_{S T} & =\mathbf{V}_{S T} \mathbf{z}_{S T} \\
\mathbf{D} \dot{\mathbf{y}}_{T} & =\mathbf{F}_{s t a t}-\mathbf{C} \mathbf{y}_{T} .
\end{aligned}
$$

Thus, the vehicle dynamics is fully characterized by the system of 24 first-order ODEs comprising the vehicle body and the axles, (5-a) and (5-b). Eight ODEs (5-g) describe the lateral and longitudinal deviations of the tires by means of spring and damper elements. The vertical deformations of the tires are covered by (5-a). The dynamic model of the drive train consists of 19 ODEs, (5-c) and (5-d), including four equations governing the angular wheel speeds. Five additional ODEs account for the dynamics of the steering system (5-e) and (5-f). Couplings between the separate systems occur via the generalized forces and torques $Q_{B V}$. Wind forces and moments are considered as additional forces applied to the multibody system of the vehicle [Chucholowski et al., 1999; Vögel et al., 2003]. 
The tire forces have a significant impact on the dynamical behavior of a vehicle. The semi-empirical tire model that is used here describes the behavior of a real tire accurately [Chucholowski et al., 1999; Vögel et al., 2003]. About 80 parameters which can be measured or estimated enter the model for each tire in veDYNA. The model covers different driving situations, including effects at the driving limits such as sliding and spinning. The actual tire model is selected online depending on the respective road and weather conditions.

Due to the stiffness of the ODE system (5-a)-(5-g) its numerical integration is carried out recursively with a semi-implicit one-step Euler scheme using a constant step size [Chucholowski et al., 1999; Vögel et al., 2003]. In particular, the integration method makes efficient use of the special block structure of the ODEs. It turns out that a fast and stable solution is possible in real-time on recent PC hardware.

For a realistic implementation of virtual test-drives by numerical simulation, additionals models for the driver and the road are required [Chucholowski et al., 1999; Vögel et al., 2003; Butz and von Stryk, 2005].

\subsubsection{Nonlinear Single Track Vehicle Model} The vehicle dynamics model should also take into account the particular properties of the ERF shock absorbers. Substitution of the commonly used linear damping behavior within the quarter and half car models of Sect. 2.2.1 by a more realistic dynamic model of the ERF damping characteristic (Sect. 2.1) yields a nonlinear system of differential equations. This may be extended by further substitutions such as nonlinear models for the tires. Such a more realistic model upgrade of a linear half car model can be used for calculation of optimal controls and trajectories, e. g., for laptime optimization [Butz and von Stryk, 2005; Vögel et al., 2003]. Specially calculated damping controls take into account pitch motions which are significant for time shifted perturbations on front and rear wheels.

\subsection{Objectives for Comfort and Safety}

Two primary objectives for a vehicle ride with semiactive suspension are ride safety and ride comfort. For both, mathematical models must be provided.

Basic and Parameterized Cost Functionals The characterization of safety in the vehicle dynamics depends primarily on wheel loads. High loads have greater longitudinal and lateral transmission forces between the wheels and the ground. On the contrary, small wheel loads can cause the loss of controllability of the vehicle. Larger magnitudes for the roll and pitch angles will indirectly influence ride safety as the magnitude of the wheels' contact forces might reach zero causing lift-off. The comfort of a ride can be of almost equal importance to passengers than safety. Comfort is mainly characterized by the accelerations of the vehicle body, often called the sprung mass contrary to the wheel body, which in this sense is called the unsprung mass. With respect to the vertical vehicle dynamics models (cf. Section 2.2.1), vertical accelerations are treated here. For models with a higher level of detail, angle accelerations of pitch and roll motions may also be considered. Altogether a performance index consisting of a weighted sum of various criteria of safety and comfort may be used, e.g.,

$$
\begin{aligned}
& \min _{\mathbf{u}} \int_{0}^{t_{f}} L(\mathbf{x}(t), \mathbf{u}(t)) \mathrm{d} t, \quad \text { where } \\
& L(\mathbf{x}, \mathbf{u})=\mu_{\text {safety }} \underbrace{\left[\sum_{i=f, r}\left[\frac{F_{\text {dyn.load }, i}}{F_{\text {stat.load }, i}}\right]^{2}+\left[\frac{x_{\theta}}{x_{\theta, \max }}\right]^{2}\right]}_{\text {safety criteria }} \\
&+\mu_{\text {comfort }} \underbrace{\left[\left[\frac{\ddot{x}_{s}}{\ddot{x}_{s, \text { max }}}\right]^{2}+\left[\frac{\ddot{x}_{\theta}}{\ddot{x}_{\theta, \text { max }}}\right]^{2}\right]}_{\text {comfort criteria }} . \text { (6) }
\end{aligned}
$$

with $F_{\text {stat.load }, i}=g\left(m_{\mathrm{s}}+m_{i}\right), \quad F_{\text {dyn.load }, i}=$ $-\left(k_{\mathrm{R}_{i}} x_{\mathrm{R}_{i}}+c_{\mathrm{R}_{i}} \dot{x}_{\mathrm{R}_{i}}\right), i=\mathrm{f}, \mathrm{r}$. For an example of the state and control variables $\mathbf{x}$ and $\mathbf{u}$, we refer to the half car model of Fig. 3. For a full vertical vehicle dynamics model one can extend the functional by variables depending on the second angle. The variables $F_{\text {dyn.load }, i}, \ddot{x}_{\mathrm{s}}, x_{\theta}, \ddot{x}_{\theta}$ denote the deviation from the corresponding stationary value.

The weights $\mu_{\diamond} \geq 0$ have to be chosen properly, i.e., depending on the purpose of investigation. For example, their actual value may depend on the actual driving situation where either comfort or safety may be more desirable. Fig. 4 shows suspension behavior for different optimal semi-active damping with respect to either only safety or comfort. Here, one purpose is to demonstrate the antagonistic character of the two goals. Optimized safety (a) leads to fast regulation of the oscillations under high accelerations of the vehicle body, whereas optimized comfort (c) yields almost decoupled motions of vehicle and wheel body under very low accelerations of the sprung mass and high frequencies of the unsprung mass. Please note the time interval with no contact of the tire with the ground in Fig. 4 (c).

Alternative Formulations Evidently it is not possible to simultaneously ensure a maximum value for the respective cost functionals corresponding to both safety and comfort by choosing particular weights of the cost functional (6). Another approach to handle this problem is to optimize one of the objectives and to restrict the other one to a suitable bound

$$
\min \int L_{\text {comfort }} \text { s.t. } \int L_{\text {safety }} \leq L_{\text {safety, } \max }
$$

and vice versa. This approach usually yields satisfactory results. A third approach is to maximize the distance from each objective from an respective upper bound using a positive slack variable $\sigma$

$$
\max \sigma \quad \text { s.t. } \quad \int L_{i} \leq L_{i, \max }-\sigma, \quad i=1,2 .
$$


(a)
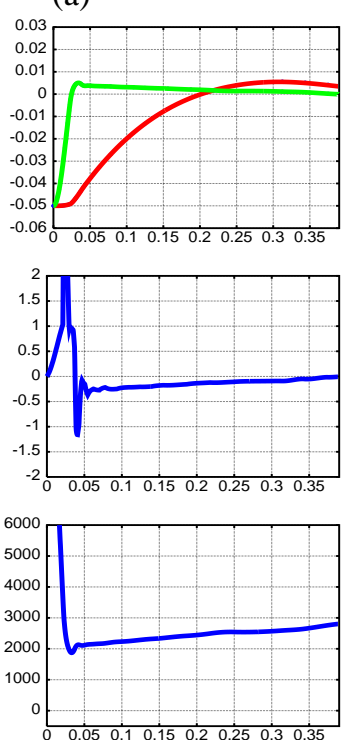

(c)
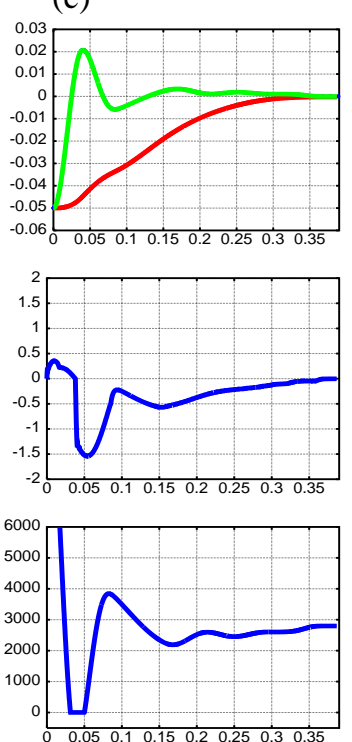

(b)
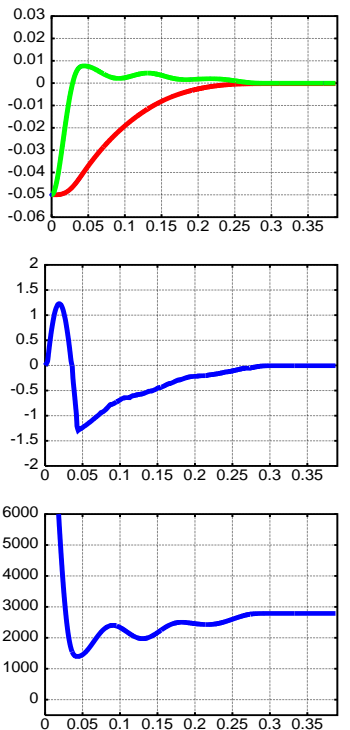

(d)
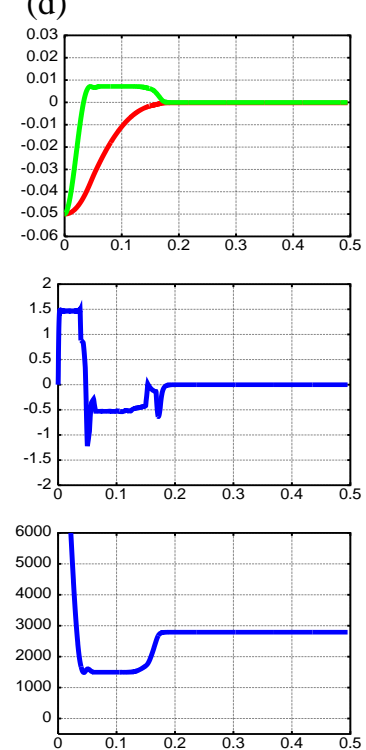

Figure 4. The results for optimal semi-active damping control of a quarter car model (Sect. 2.2.1) for different objectives are displayed which have been obtained using the method of Sect. 4.1 and $t_{f}=0.4 \mathrm{~s}$. The objectives used in the columns are (a) optimal safety, comfort ignored, (b) optimal comfort with constrained wheel load as in Eq. (7), (c) optimal comfort, safety ignored, (d) objective of Eq. (8) with a slack variable. The rows in each column depict in the first row the wheel displacement $x$ (bright, green line) and the vehicle body displacement $x_{s}$ (dark, red line), in the second row the vehicle body acceleration $\left[\mathrm{m} / \mathrm{s}^{2} / \mathrm{g}\right]$, and in the third row the wheel load $F_{R}[\mathrm{kN}]$.

Fig. 4 shows the behavior of the state variables of a quarter car model for the resulting optimal controlled semi-active suspension which has been computed using the method of Sect. 4.1. The last optimization configuration using a slack variable yields high gain damping.

However, the weight selection may depend on the particular vehicle or ride purpose. Active suspensions for race or sports cars, ambulance vehicles or passenger cars have to be designed by different requirements.

\section{Optimal and Robust Optimal Linear Quadratic Controls}

The goal of the control problem for controllable ERFshock absorbers integrated into a semi-active vehicle suspension is a combination of optimal safety and optimal comfort of the ride. In comparison to a fixed damping characteristic or a manual selection between a few, alternative characteristics, the new technology of ERFshock absorbers permits continuous control. This suggests the calculation of suitable (if possible optimal) controls taking into account the possible nonlinear dynamics of the vehicle and of the damper (Section 2.2) and further constraints.

\subsection{State Feedback Control Based on LQR and $\mathbf{H}^{\infty}$ Techniques}

In order to investigate the capability of semi-active damping with ERF shock absorbers, state feedback controls can be derived for various linear vehicle dynamics models (cf. Sect. 2.2) written as

$$
\dot{\mathbf{x}}=\underbrace{\left(\begin{array}{cc}
\mathbf{0}_{n_{q}, n_{q}} & \mathbf{I}_{n_{q}, n_{q}} \\
\mathbf{M}^{-1} \mathbf{A}_{\mathrm{k}} \mathbf{M}^{-1} \mathbf{A}_{\mathrm{c}}
\end{array}\right)}_{\mathbf{A}} \mathbf{x}+\underbrace{\left(\begin{array}{c}
\mathbf{0}_{n_{q}, n_{u}} \\
\mathbf{M}^{-1} \mathbf{B}_{\mathrm{m}}
\end{array}\right)}_{\mathbf{B}} \mathbf{u}
$$

with the state $\mathbf{x}=(\mathbf{q}, \dot{\mathbf{q}}) \in \mathbb{R}^{n_{x}}$ and the control $\mathbf{u} \in \mathbb{R}^{n_{u}}$, where $\mathbf{q} \in \mathbb{R}^{n_{q}}$ denotes the generalized coordinates, e.g., $\mathbf{q}=\left(x_{s}, x\right)$ for the quarter car model and $\mathbf{q}=\left(x_{s}, x_{\theta}, x_{r}, x_{f}\right)$ for the half car model of Sect. 2.2.1. The further elements of Eq. (9) denote the zero matrix $\mathbf{0}$, the unit matrix $\mathbf{I}$, the mass matrix $\mathbf{M}$, the stiffness matrix $\mathbf{A}_{\mathrm{k}}$, the damping matrix $\mathbf{A}_{\mathrm{c}}$, the control matrix $\mathbf{B}_{\mathrm{m}}$. The state $\mathbf{x}$ is controlled by $\mathbf{u}$. $n_{\diamond}$ denote their dimensions, $\diamond=x, q$, or $u$.

As the linear quadratic cost function

$$
J[\mathbf{u}]=\int_{0}^{\infty} \mathbf{x}^{\mathrm{T}} \mathbf{Q} \mathbf{x}+2 \mathbf{u}^{\mathrm{T}} \mathbf{S} \mathbf{x}+\mathbf{u}^{\mathrm{T}} \mathbf{R} \mathbf{u} \mathrm{d} t
$$

a weighted criterion for safety and comfort is chosen which consists of oscillations of contact forces representing the fundamental safety criterion, and vehicle body accelerations representing a relevant comfort criterion

$$
\left.\begin{array}{rl}
\mathbf{Q}= & \mu_{\text {comfort }} \sum_{\begin{array}{c}
\text { states } \\
\text { vehicle body }
\end{array}} \frac{1}{\ddot{x}_{i, \max }^{2}} \mathbf{A}^{\mathrm{T}} \mathbf{e}_{i} \mathbf{e}_{i}^{\mathrm{T}} \mathbf{A} \\
& +\mu_{\text {safety }} \sum_{\text {wheels }}\left[\frac{k_{\mathrm{R} i}}{F_{\text {stat.load }, i}}\right]^{2}\left(\mathbf{e}_{i} \mathbf{e}_{i}^{\mathrm{T}}\right) \\
& +\ldots+\sum_{\text {states }} \mu_{i, \text { state }} \frac{1}{x_{i, \max }^{2}}\left(\mathbf{e}_{i} \mathbf{e}_{i}^{\mathrm{T}}\right) \\
\mathbf{R}= & \mu_{\text {comfort }} \sum_{\text {states }} \frac{1}{\ddot{x}_{i, \max }^{2}} \mathbf{B}^{\mathrm{T}} \mathbf{e}_{i} \mathbf{e}_{i}^{\mathrm{T}} \mathbf{B} \\
& +\mu_{\text {costs }} \sum_{\text {wheels }}^{\text {vehicle body }} \frac{1}{u_{i, \max }} \mathbf{B}^{\mathrm{T}} \mathbf{e}_{i} \mathbf{e}_{i}^{\mathrm{T}} \mathbf{B} \\
\mathbf{S}= & \mu_{\text {comfort }} \sum_{\text {states }} \frac{1}{\ddot{x}_{i, \max }^{2}} \mathbf{B}^{\mathrm{T}} \mathbf{e}_{i} \mathbf{e}_{i}^{\mathrm{T}} \mathbf{A}
\end{array}\right\}
$$


with weights $\mu_{\diamond} \geq 0$. The sums over the states of the vehicle body take into account vertical oscillations as well as angular oscillations if they appear in the model. The sums over the states of the wheels include all considered wheel oscillations. To ensure existence of the linear optimal control additional quadratic penalty terms due to the state and control variables have to be taken into account. It is well known that the optimal feedback control can now be obtained as

$$
\mathbf{u}^{*}=\mathbf{R}^{-1}\left(\mathbf{S}+\mathbf{B}^{\mathrm{T}} \mathbf{P}\right) \mathbf{x}
$$

with $\mathbf{P}$ as the solution of the algebraic Riccati equation (cf., e.g., [Dorato, Abdallah and Cerone, 1994])

$$
\mathbf{A}^{\mathrm{T}} \mathbf{P}+\mathbf{P A}+\mathbf{Q}-\left(\mathbf{P B}+\mathbf{S}^{\mathrm{T}}\right) \mathbf{R}^{-1}\left(\mathbf{B}^{\mathrm{T}} \mathbf{P}+\mathbf{S}\right)=\mathbf{0}
$$

corresponding to the cost functional of Eq. (10). The optimal solution exists, if the system is stabilizable, $\mathbf{R}$ is positive definite, and $\mathbf{Q}-\mathbf{S}^{\mathrm{T}} \mathbf{R}^{-1} \mathbf{S}$ is positive semi definite. The last two properties hold, if the objective (10) contains the quadratic penalty terms of the state and control variables as in Eq. (11).

Furthermore, we may consider robust-optimal controls, regarding unknown perturbations w, e.g. by the ground. Then, the dynamic equations (9) include a perturbation term

$$
\dot{\mathbf{x}}=\mathbf{A} \mathbf{x}+\mathbf{B u}+\underbrace{\left(\begin{array}{c}
\mathbf{0}_{n_{q}, n_{w}} \\
\mathbf{M}^{-1} \mathbf{D}_{\mathrm{m}}
\end{array}\right)}_{\mathbf{D}} \mathbf{w}
$$

with the perturbation matrix $\mathbf{D}_{\mathrm{m}}$. Perturbations include impacts caused by an uneven ground as well as forces and moments acting on the vehicle body caused by driving maneuvers. Let us now consider the augmented objective

$$
J_{\gamma}[\mathbf{u}, \mathbf{w}]=\int_{0}^{\infty} \mathbf{x}^{\mathrm{T}} \mathbf{Q} \mathbf{x}+2 \mathbf{u}^{\mathrm{T}} \mathbf{S} \mathbf{x}+\mathbf{u}^{\mathrm{T}} \mathbf{R} \mathbf{u}
$$

with the attenuation bound $\gamma>0$. Then the robustoptimal $\mathrm{H}^{\infty}$ control is the saddle-point solution of a dynamic game where $\mathbf{u}$ is the player minimizing and $\mathbf{w}$ the player maximizing the objective. It exists, if the solution $\mathbf{P}_{\gamma} \in \mathbb{R}^{n_{x} \times n_{x}}$ of the augmented algebraic Riccati equation

$$
\left.\begin{array}{r}
\mathbf{A}^{\mathrm{T}} \mathbf{P}_{\gamma}+\mathbf{P}_{\gamma} \mathbf{A}+\mathbf{Q}-\left[\mathbf{P}_{\gamma}[\mathbf{B} \mathbf{D}]+\left[\mathbf{S}^{\mathrm{T}} \mathbf{0}^{\mathrm{T}}\right]\right] \\
\cdot\left[\begin{array}{cc}
\mathbf{R}^{-1} & \mathbf{0} \\
\mathbf{0} & -\frac{1}{\gamma^{2}}
\end{array}\right]\left[\left[\begin{array}{l}
\mathbf{B}^{\mathrm{T}} \\
\mathbf{D}^{\mathrm{T}}
\end{array}\right] \mathbf{P}_{\gamma}+\left[\begin{array}{l}
\mathbf{S} \\
\mathbf{0}
\end{array}\right]\right]=\mathbf{0}
\end{array}\right\}
$$

exists (cf., e.g. [Basar and Bernhard, 1991; Basar and Olser, 1995]). The robust-optimal control $u_{\text {rob }}^{*}$ then follows from Eq. (12).
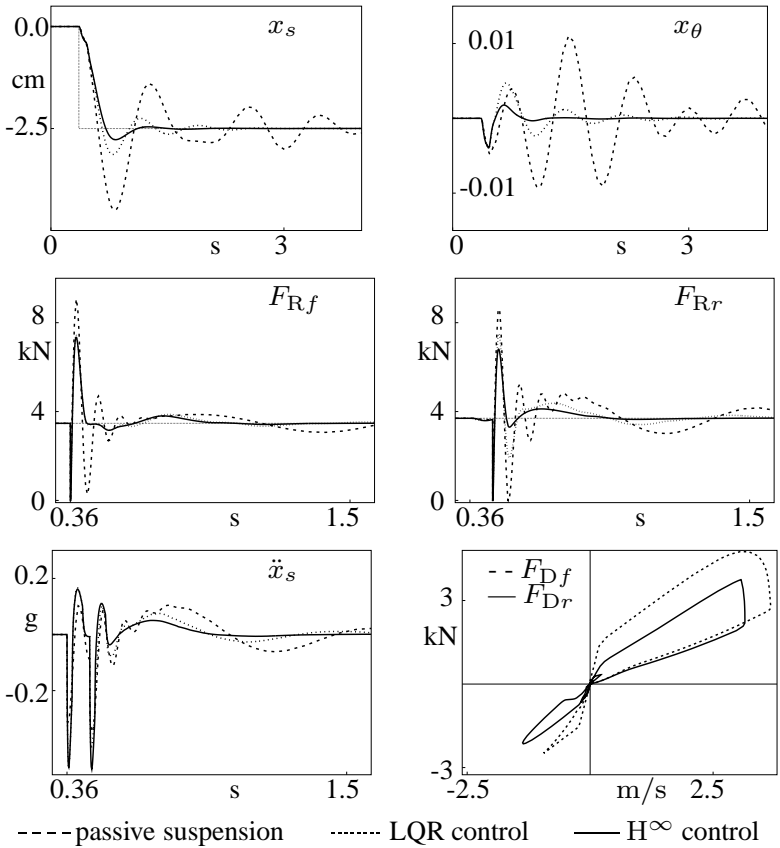

Figure 5. Comparison of some of the state variables of a (linear) single track model for a ride over a step of $2.5 \mathrm{~cm}$ height at a speed of $100 \mathrm{~km} / \mathrm{h}$ for LQR- and $\mathrm{H}^{\infty}$-controlled suspensions.

For $\gamma>0$ and optimal $\mathbf{u}^{0}=\mathbf{u}^{0}(\mathbf{w})$ we have $J\left[\mathbf{u}^{0} ; \mathbf{w}\right] /\|\mathbf{w}\|^{2} \leq \gamma^{2}, \forall \mathbf{w}$, i.e. the disturbance of the system is bounded. The optimal disturbance attenuation is related to a lower bound $\gamma^{*}$, which can only be calculated iteratively. Since the existence of an optimal controller for the infimum value $\gamma^{*}$ is not guaranteed, suboptimal solutions for $\gamma=\gamma^{*}+\varepsilon_{j}$ may be computed iteratively for a series of decreasing "small" $\varepsilon_{j}>0$ [Helton and James, 1999; Hoppe et al., 2000].

\subsection{Semi-Active Control of ERF Shock Absorber}

Please note that the linear-quadratic optimal control merely provides an optimal damping force, but not an optimal damping rate or optimal electrical field to be applied at the valve within the semi-actively working shock absorber (Sect. 2.1). Such an active damping system would provide (bounded) forces like an actuator. Whereas semi-active shock absorbers only control damping forces, always regarding the direction of motion of the damping element. They can not add but only extract energy from the system.

A practical semi-active damping rate is now predicted on the basis of the optimal (or robust-optimal) damping force $F_{\mathrm{D} \text {,opt }}$ obtained in Sect. 3.1, e.g., by the LQR or $\mathrm{H}^{\infty}$ approach. For a quarter car model we have $F_{\mathrm{D}, \text { opt }}=\mathbf{u}^{*}$. Using a heuristic compensation regulator, we apply with $F_{\mathrm{D} \text {,cur }}, u_{\mathrm{D} \text {,cur }}$ as the current force a current control at each ERF damper

$u_{\mathrm{D}, \text { appl }}=\left\{\begin{array}{l}u_{\min }, \text { for } F_{\mathrm{D}, \text { opt }} F_{\mathrm{D}, \text { cur }}<0 \\ \min \left(u_{\max }, \max \left\{u_{\min }, u_{\mathrm{D}, \text { cur }}+\right.\right. \\ \left.\left.\quad\left(\left|F_{\mathrm{D}, \text { opt }}\right|-\left|F_{\mathrm{D}, \mathrm{cur}}\right|\right) * K\right\}\right), \text { otherwise }\end{array}\right.$ 
which is similar to a "clipped optimal" control algorithm as suggested by [Spencer et al., 1996]. $F_{\mathrm{D} \text {,cur }}$ is assumed to be known and the constant $K$ depends on the scaling of $u_{\mathrm{D}}$ and the selected sampling rate.

\subsection{Numerical Results}

In Fig. 5 the results are depicted for some of the state variables of a (linear) single track model for a ride over a step of $2.5 \mathrm{~cm}$ height at a speed of $100 \mathrm{~km} / \mathrm{h}$ for semiactive suspension with $\mathrm{LQR}$ or $\mathrm{H}^{\infty}$ control resulting for a large weight of comfort and compared with a passive suspension. Depending on the value of $\gamma$ the $\mathrm{H}^{\infty}$ control performance ranges from close to the $\mathrm{LQR}$ control to a high level of robust optimality being capable of compensating large disturbances $\mathbf{w}$.

\subsection{Validation by Software-in-the-Loop Simula- tion}

An ISO double lane change maneuver for a full motor vehicle dynamics model of a midclass car (Sect. 2.2.2) is considered. The target trajectory of the vehicle's center of mass corresponds to an optimized driving control maneuver with respect to minimum time [Vögel et al., 2003] on an even road. In order to test the vehicle's capabilities in an extreme situation, the same ride is now considered subject to perturbations resulting from a wavy road surface with amplitudes between 20 and $40 \mathrm{~cm}$. The resulting driving conditions are apparently unsafe, because the vehicle skids off the road (see Fig. 6, bright line). Repetition of the maneuver with semi-actively $\mathrm{H}^{\infty}$-controlled ERF shock absorbers with equally weighted comfort and safety, shows the benefit of robustness. Using the same guidance control for the vehicle, it successfully follows the same set point trajectory but now on a very rough road.

\section{Optimal Semi-Active Control Based on Nonlin- ear Dynamics}

4.1 Nonlinear Deterministic Optimal Trajectories Direct transcription methods have been developed very successfully during the last decade and promise high flexibility and robustness when solving general optimal control problems with a Mayer type objective

$$
\left.\begin{array}{rl}
\min J[\mathbf{u}] & =\Phi\left(\mathbf{x}\left(t_{\mathrm{f}}\right), t_{\mathrm{f}}\right) \\
\dot{\mathbf{x}}(t) & =\mathbf{f}(\mathbf{x}(t), \mathbf{u}(t), t), \quad 0 \leq t \leq t_{\mathrm{f}} \\
0 & =\mathbf{r}\left(\mathbf{x}(0), \mathbf{x}\left(t_{\mathrm{f}}\right), t_{\mathrm{f}}\right) \\
0 & \leq \mathbf{g}(\mathbf{x}(t), \mathbf{u}(t), t)
\end{array}\right\}
$$

numerically to low or moderate accuracies, e.g., [Betts, 2001]. In the direct collocation method [von Stryk, 2001] a discretization of $\mathbf{x}$ by piecewise cubic Hermite polynomials $\tilde{\mathbf{x}}(t)=\sum_{k} \alpha_{k} \hat{\mathbf{x}}_{k}(t)$ and of $\mathbf{u}$ by piecewise linear functions $\tilde{\mathbf{u}}(t)=\sum_{k} \beta_{k} \hat{\mathbf{u}}_{k}(t)$ is applied [von Stryk, 2003] on a discretization grid $t_{a}=t_{1}<$ $t_{2}<\ldots<t_{n_{t}}=t_{f}$, see Fig. 7. The equations of motions (17) are pointwise fulfilled at the grid points and
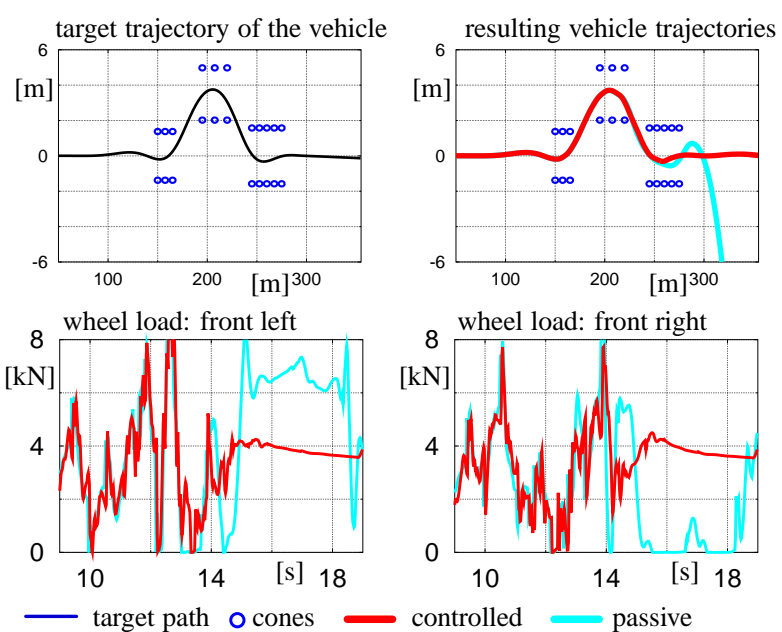

Figure 6. The double lane change maneuver (path through the cones depicted in the upper row) on a very uneven road, simulated with the full vehicle dynamics model of a passenger car in real-time. The car with a conventional passive suspension skids off the road. Whereas the car with an (sub-) optimally controlled semi-active suspension follows the target trajectory for the vehicle's center of mass. The figures in the lower row show wheel loads on the right and left front wheels of the full vehicle dynamics model for a passive and a clipped semi-actively controlled ride during the critical phase of the maneuver.

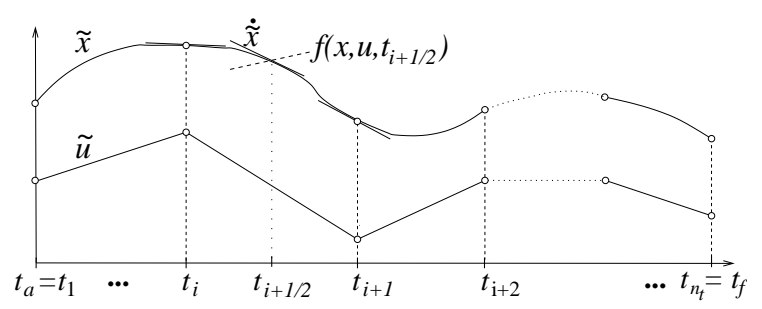

Figure 7. Direct collocation parameterization of continuous state and control variables.

at their respective midpoints resulting in a set of nonlinear NLP equality constraints $\mathbf{a}(\mathbf{y})=0$ (collocation). Any control or state variable inequality constraints are to be satisfied at the grid points resulting in set of nonlinear NLP inequality constraints $\mathbf{b}(\mathbf{y}) \geq 0$. Here, $\mathbf{y}$ denotes the $n_{y}$ parameters of the parameterization. The resulting nonlinearly constrained optimization problem (NLP) basically reads as

$$
\left.\begin{array}{c}
\min _{\mathbf{y}} \Phi(\mathbf{y}) \text { subject to } \mathbf{a}(\mathbf{y})=0, \mathbf{b}(\mathbf{y}) \geq 0 \\
\mathbf{y}=\left(\alpha_{1}, \alpha_{2}, \ldots, \beta_{1}, \beta_{2}, \ldots, t_{f}\right)^{T} .
\end{array}\right\}
$$

Please note that an objective involving an integral term as in Eq. (10) can easily be transformed into a problem with a Mayer-type objective as in Eq. (17) introducing one additional state equation. The gradients and Jacobians of the NLP exhibit a sparse structure which can be exploited by the large-scale SQP method SNOPT [Gill, Murray and Saunders, 2002] resulting in computational speed-ups by two orders of magnitude [von Stryk, 2003] compared to standard NLP solvers. Usually a sequence of related NLPs with refined time grids 
is solved successively to obtain a good approximation. In addition, the method provides reliable estimates of the adjoint or costate variables $\boldsymbol{\lambda}$ of the optimal control problem.

\subsection{Extension to Nonlinear Robust Optimal Semi- Active Control Strategies}

Control of shock absorbers must account for unknown disturbances. For vehicle rides in particular these are changes of the road height or inertial forces and moments caused by unexpected driving maneuvers like braking, accelerating etc. In recent years $H^{\infty}$ control theory gained increased scientific interest (cf. [Helton and Ball, 1989; Helton and Ball, 1995; Basar and Bernhard, 1991; Kwakernak, 1985; Schaft, 1991; Schaft, 1996; Soravia, 1996; Helton and James, 1999]). Here robust-optimal controls optimize the gain of the system under worst excitations. Consider the extended nonlinear state space system and optimal control problem with suitable functions $\mathbf{f}, l$

$$
\left.\begin{array}{rl}
\dot{\mathbf{x}} & =\mathbf{f}(\mathbf{x}, \mathbf{u}, \mathbf{w}), \quad \mathbf{x}\left(t_{0}\right)=\mathbf{x}_{0}, \\
J(\mathbf{u}, \mathbf{w}) & =\int_{t_{0}}^{t_{\mathrm{f}}} l(\mathbf{x}, \mathbf{u}, \mathbf{w}) \mathrm{d} t \rightarrow \min !
\end{array}\right\}
$$

where $\mathbf{u} \in U \subset \mathbb{R}^{n_{u}}, \mathbf{w} \in L_{2}\left(\mathbb{R}^{n_{w}}\right)$. In order to treat robustness of nonlinear systems, suitable terms of stability have to be defined. The system (19) with fixed control $\overline{\mathbf{u}}=\overline{\mathbf{u}}(\mathbf{x})$ and operator $\mathbf{T}_{\overline{\mathbf{u}}}: \mathbf{w} \mapsto \mathbf{x}$ is said to be finite-gain L-stable if there are $\gamma, \beta \geq 0$ with

$$
\left\|\mathbf{T}_{\overline{\mathbf{u}}}(\mathbf{w})\right\|_{L_{2}} \leq \gamma\|\mathbf{w}\|_{L_{2}}+\beta, \quad \forall \mathbf{w} \in L_{2}\left(\mathbb{R}^{n_{w}}\right)
$$

The value $\gamma^{*}=\inf \{\gamma \mid$ Eq. (20) is satisfied $\}$ is the gain, and in case of linear-quadratic problems $\gamma^{*}$ is the $\mathrm{H}^{\infty}$-norm of the system. If Eq. (20) is satisfied for system (19) for any $\bar{\gamma}$ by control $\overline{\mathbf{u}}$, then the control $\overline{\mathbf{u}}$ is called robust-optimal with respect to the attenuation level $\bar{\gamma}$. Note that in this sense it is not necessary to find robust-optimal controls with respect to the infimum attenuation level $\gamma^{*}$. It should be mentioned, that from an engineering point of view, robust controls related to attenuation levels close to $\gamma^{*}$ very often are high gain controllers (cf. [Basar and Bernhard, 1991]). Hence a value $\gamma$ close to $\gamma^{*}$ will be a favorable compromise as $\gamma^{*}$ may be difficult or even impossible to determine numerically.

The required robust-optimal control has to satisfy both, finite-gain stability and stability of the undisturbed system. Therefore dissipative systems are investigated (cf. [Helton and Ball, 1989; Schaft, 1996]). The dynamic system (19) is dissipative with respect to a given supply rate $s(\mathbf{w}, \mathbf{v}) \in \mathbb{R}$ and system output function $\mathbf{v}(\mathbf{x}, \mathbf{u}, \mathbf{w}) \in \mathbb{R}^{n_{v}}$, if there exists an energy function $S(\mathbf{x}) \geq 0$, such that for all $\mathbf{x}\left(t_{0}\right)=\mathbf{x}_{0}$ and $t_{1} \geq t_{0}$ and for all $\mathbf{w} \in L_{2}\left(\mathbb{R}^{n_{w}}\right)$

$$
S\left(\mathbf{x}\left(t_{1}\right)\right) \leq S\left(\mathbf{x}\left(t_{0}\right)\right)+\int_{t_{0}}^{t_{1}} s(\mathbf{w}, \mathbf{v}) \mathrm{d} t
$$

holds with $\mathbf{x}\left(t_{1}\right)=\mathbf{x}\left(\mathbf{t}_{1} ; \mathbf{x}_{0}, t_{0}, \mathbf{w}\right)$; i.e. the dissipation inequality (21) has to be satisfied along all trajectories with free initial value $\mathbf{x}_{0}$ (cf. [Willems, 1972]).

With particular supply rate $s(\mathbf{w}, \mathbf{v})=\gamma^{2}\|\mathbf{w}\|^{2}-$ $\|\mathbf{v}\|^{2}$ a dissipative system yields finite-gain stability, $\mathrm{cf}$. [Schaft, 1996]. Stability of the undisturbed system was shown in [Schaft, 1996] assuming that a continuously differentiable energy function exists; for the discontinuous case cf. [Soravia, 1996].

Considering the differential dissipation inequality with $l=\|\mathbf{v}\|^{2}$, the solution of the saddle-point problem $\min _{\mathbf{u} \in U} \max _{\mathbf{w} \in L_{2}} \partial S / \partial \mathbf{x} \mathbf{f}(\mathbf{x}, \mathbf{u}, \mathbf{w})+l(\mathbf{x}, \mathbf{u}, \mathbf{w})-\gamma^{2}\|\mathbf{w}\|^{2} \leq 0$

provides existence of an energy function and hence dissipativity, cf. [Helton and Ball, 1989]. The saddlepoint $\left(\mathbf{u}^{*}, \mathbf{w}^{*}\right)$ of Eq. (22) minimizes the functional of (19) under the dissipation constraint with respect to disturbance attenuation bound $\gamma$.

Equation (22) is of Hamilton-Jacobi-Isaacs type (cf. [Isaacs, 1967; Basar and Bernhard, 1991]). The saddlepoint $\left(\mathbf{u}^{*}, \mathbf{w}^{*}\right)$ relates to the extended functional

$$
J_{\gamma}(\mathbf{u}, \mathbf{w})=\int_{t_{0}}^{t_{\mathrm{f}}} l(\mathbf{x}, \mathbf{u}, \mathbf{w})-\gamma^{2}\|\mathbf{w}\|^{2} \mathrm{~d} t
$$

and dynamic equations (19). Note that necessarily $J_{\gamma}\left(\mathbf{u}^{*}, \mathbf{w}^{*}\right) \leq 0$ follows from (21) and has to be tested for any numerical solution of the saddlepoint problem. If the inequality is not fulfilled, attenuation bound condition (20) is violated.

Necessary conditions for a saddlepoint follow from Isaacs minmax principle (cf. [Isaacs, 1967; Basar and Olser, 1995]), supposing that the minimization and maximization in (22) can be exchanged. With $H(\mathbf{x}, \boldsymbol{\lambda}, \mathbf{u}, \mathbf{w})=\boldsymbol{\lambda}^{\mathrm{T}} \mathbf{f}(\mathbf{x}, \mathbf{u}, \mathbf{w})+l(\mathbf{x}, \mathbf{u}, \mathbf{w})-\gamma^{2}\|\mathbf{w}\|^{2}$ as the Hamiltonian function, optimal feedback controls must satisfy pointwise

$$
\left.\begin{array}{rl}
\mathbf{u}^{*} & =\underset{\mathbf{u} \in U}{\operatorname{argmin}} H(\mathbf{x}, \boldsymbol{\lambda}, \mathbf{u}, \mathbf{w}), \\
\mathbf{w}^{*} & =\underset{\mathbf{w} \in L_{2}}{\operatorname{argmax}} H(\mathbf{x}, \boldsymbol{\lambda}, \mathbf{u}, \mathbf{w}) \cdot
\end{array}\right\}
$$

Characteristic solutions of the partial differential equation (22) are represented by time-dependent optimal trajectories $\mathbf{x}^{*}(t)$ and adjoint variables $\boldsymbol{\lambda}^{*}(t)$, satisfying the boundary value problem

$$
\left.\begin{array}{l}
\dot{\mathbf{x}}=\mathbf{f}\left(\mathbf{x}, \mathbf{u}^{*}, \mathbf{w}^{*}\right) \\
\dot{\boldsymbol{\lambda}}=-\left(\partial H\left(\mathbf{x}, \boldsymbol{\lambda}, \mathbf{u}^{*}, \mathbf{w}^{*}\right) / \partial \mathbf{x}\right)^{\mathrm{T}}
\end{array}\right\}
$$

and corresponding boundary conditions for $\mathbf{x}$ and $\lambda$. For numerical solution we use the direct collocation method described in Sect. 4.1. In the case of optimal control problems we do not need to consider the dynamic equations of the adjoint variables explicitly. 
perturbations $w$

for decreasing values of $\gamma$

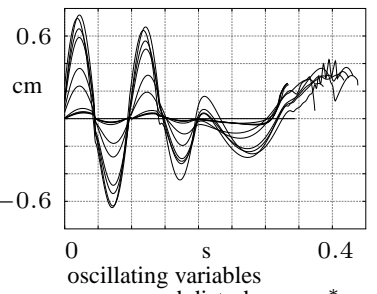

$x_{\mathrm{s}}, x_{\mathrm{R}}$ and disturbance $w^{*}$

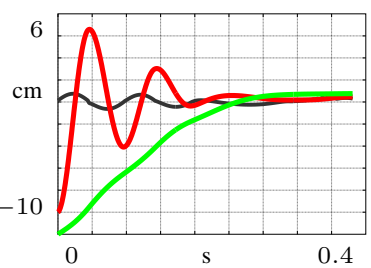

adjoint variables of $x_{\mathrm{s}}, x_{\mathrm{R}}$

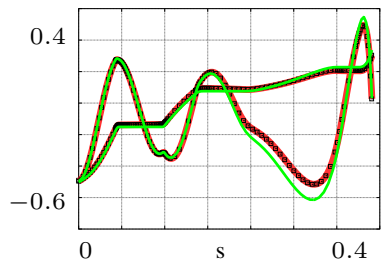

damping control $u_{c_{\mathrm{S}}}$

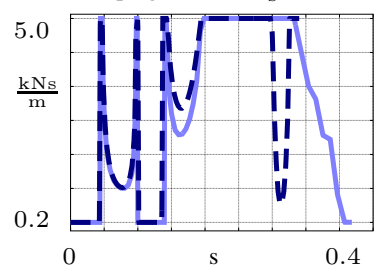

velocity variables $\dot{x}_{\mathrm{s}}, \dot{x}_{\mathrm{R}}$

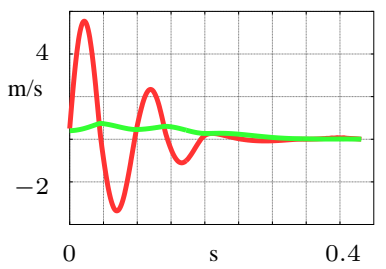

adjoint variables of $\dot{x}_{\mathrm{s}}, \dot{x}_{\mathrm{R}}$

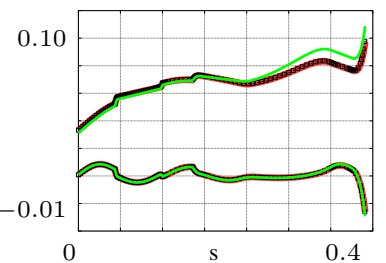

Figure 8. Numerical solutions for a robust-optimal control problem obtained by the approach outlined in Sect. 4.2. Upper left: Growth of the worst perturbation obtained for different, decreasing values of the attenuation bound $\gamma$ which displays a constant frequency. Upper right: Comparison of the damping control - solution of discretized problem with piecewise linear control approximation (solid), subsequently calculated by the minimum principle using the computed trajectories of state and adjoint variables (dashed). Please note the complex switching structure. Second row, left: Trajectories of oscillating variables $x_{\mathrm{S}}$ (light, green line) and $x_{\mathrm{R}}$ (dark, red line) compared to the calculated worst perturbation $w$ (left, black) with respect to the corresponding attenuation level $\gamma$. Second row, right: corresponding velocity variables $\dot{x}_{\mathrm{S}}, \dot{x}_{\mathrm{R}}$. Third row: Comparison of adjoint variables as estimated by the method of Sect. 4.1.

For the robust-optimal control problem (23)-(25) we have to take into account the unknown disturbance $\mathbf{w}(t)$. As originally suggested by [Horie and Conway, 2000] we discretize both, the state and adjoint differential equations (25) and minimize (23) directly for the discretized damping control $\mathbf{u}(t)$ and compute the disturbance $\mathbf{w}(t)$ from (24). Fig. 8 shows the results of such a procedure. The mentioned discretization scheme was applied to a quarter car model subject to the boundary condition from (19) and the constraints $0.2 \leq u_{c_{\mathrm{s}}} \leq 0.5$ on the damping control which subsumizes spring and damper elements.

Usually the discretization of the transformed optimal control problem with extended dynamic equations (25) provides comparatively accurate solutions for the undisturbed system and for large values of $\gamma$. Numerical difficulties arise with decreasing $\gamma \rightarrow \gamma^{*}$, possibly caused by the structure of the extended adjoint equations with respect to the transformed optimal control problem, whose solutions are supposed to be damped for large values of $\gamma$ only. In order to make a com-

promise one has to calculate with lower accuracy. An automation of so-called $\gamma$-strategies in order to find solutions for $\gamma$ close to attenuation bound $\gamma^{*}$ are possible, e.g. by a bisection or continuation method. The desired accuracy may be determined by a consistency check between the calculated discretized adjoint variables and the according estimates by the discretization method (cf. Fig. 8, third row). Please note, that the chosen discretization and direct optimization scheme enables the computation of controls with a priori unknown complex switching structures. On the other hand new difficulties arise in the context of singular surfaces, which play an important role in differential game theory [Isaacs, 1967]. Particularly surfaces with discontinuities of the value function, so-called barriers, are not detected by the proposed numerical method. Hence one has to check a posteriori if calculated trajectories intersect such barriers [Breitner, Pesch and Grimm, 1993].

\section{Conclusions}

The problem of optimal semi-active suspension of vehicles using the new technology of electrorheological fluid dampers has been investigated. For the formulation of the corresponding optimal control problems, several models of the vehicle dynamics, of the ERF shock absorber dynamics and of objectives for safety and comfort have been presented and investigated. Reduced, linear vehicle dynamic models permit the application of LQR and $\mathrm{H}^{\infty}$ control techniques which provide an optimal and real-time capable feedback control for the reduced model. The resulting controls are only suboptimal with respect to the full scale vehicle dynamics model but provide remarkable improvements over passive suspensions as has been demonstrated in numerical experiments. Furthermore, direct transcription methods can deal with general, nonlinear dynamic models and constraints, but only provide optimal openloop state and control trajectories which may serve for a numerical feedback synthesis. Finally, it has been outlined how the nonlinear $\mathrm{H}^{\infty}$ control problem can be formulated as a nonlinear zero-sum dynamic game problem. Candidates for robust optimal trajectories for semi-active suspension can then be computed numerically by a mixed direct-indirect transcription approach as has been demonstrated in an example. Furthermore, different approaches to consider objectives for comfort and safety with their antagonistic properties in a single objective have been investigated.

\section{References}

Alleyne, A. and Hedrick, K.J. (1995). Nonlinear adaptive control of active suspensions. IEEE Transactions on Control Systems Technology, 3(1), pp. 94-101.

Ball, J.A., and Helton, J.W. (1989). $\mathrm{H}_{\infty}$ control for nonlinear plants: connections with differential games. Proc. 28th Conf erence on Decision and Control, Tampa, Florida, pp. 956-962. 
Ball, J.A., and Helton, J.W. (1995). Viscosity solutions of Hamilton-Jacobi equations arising in nonlinear $\mathrm{H}_{\infty}$ control. Journal of Mathematical Systems, Estimation, and Control, 6(1).

Basar, T. and Bernhard, P. (1991). $H^{\infty}$-Optimal Control and Related Minimax Design Problems. A Dynamic Game Approach. Birkhäuser, Boston, Basel, Berlin.

Basar, T. and Olsder, G.J. (1995). Dynamic Noncooperative Game Theory. Academic Press, London, San Diego, New York.

Betts, J.T. (2001). Practical Methods for Optimal Control Using Nonlinear Programming. Advances in Design and Control 3. SIAM. Philadelphia.

Breitner, M.H., Pesch, H.J. and Grimm, W. (1993) Complex Differential Games of Pursuit-Evasion Type with State Constraints, Part 1: Necessary Conditions for Optimal Open-Loop Strategies, Part 2: Numerical Computation of Optimal Open-Loop Strategies. J. of Optimization Theory and Applications 78:3, pp. 419441, 443-463.

Butz, T. and von Stryk, O. (2002). Modelling and simulation of electro- and magnetorheological fluid dampers. Z. Angew. Math. Mech. 82:1, pp. 3-20.

Butz, T. and von Stryk, O. (2005). Optimal control based modeling of vehicle driver properties. In Society of Automotive Engineers (SAE) 2005 World Congress, Detroit, MI, U.S.A., April 11-14, SAE Paper 2005-01-0420.

Chucholowski, C., Vögel, M., von Stryk, O. and Wolter, T.M. (1999). Real time simulation and online control for virtual test drives of cars. In: H.-J. Bungartz et al. (eds.): High Performance Scientific and Engineering Computing. Vol. 8 of Lecture Notes in CSE. Springer-Verlag, Berlin. pp. 157-166.

Dorato, P., Abdallah, C. and Cerone, V. (1994). Linear Quadratic Control: An Introduction. Prentice Hall, Englewood Cliffs, N.J.

Genta, G. (1997). Motor Vehicle Dynamics, Modelling and Simulation. Series on Advances in Mathematics for Applied Sciences 43, World Scientific, London.

Gill, P., Murray, W. and Saunders, M. (2002). SNOPT: An SQP algorithm for large-scale constrained optimization. SIAM J. Optimization, 12, pp. 979-1006.

Hać, A. (1992). Optimal linear preview control of active vehicle suspension. Vehicle System Dynamics, 21, pp. 167-195.

Helton, J.W. and James, M.R. (1999). Extending $H_{\infty}$ Control to Nonlinear Systems. SIAM, Philadelphia.

Hoppe, R.H.W., Mazurkevitch, G., Rettig, U. and von Stryk, O. (2000). Modeling, simulation, and control of electrorheological fluid devices. In: H.-J. Bungartz et al. (eds.). High Performance Scientific and Engineering Computing. Vol. 8 of Lectures on Applied Mathematics. Springer-Verlag, pp. 157-166.

Horie, K. and Conway, B.A. (2000). Collocation with Nonlinear Programming for Zero-Sum Differential Games. Manuscript, private communication by B.A. Conway in June 2000.
Isaacs, R.P. (1967). Differential Games. John Wiley \& Sons, Inc., New York.

Kortüm, W. and Lugner, P. (1998). Systemdynamik und Regelung von Fahrzeugen. Springer Verlag, Berlin.

Kwakernaak, H. (1985). Minimax frequency domain performance and robustness optimization of linear feedback systems. IEEE Transactions on Automatic Control, 30(10), pp. 994-1004.

Popp, K. and Schiehlen, W. (1993). Fahrzeugdynamik. T.B. Teubner, Stuttgart.

Rettig, U. (2003). Optimale und robust-optimale Steuerungen: Grundlagen, numerische Berechnung und Anwendung bei der semi-aktiven KfzSchwingungsdämpfung. Nr. 992, Reihe 8: Meß-, Steuerungs- und Regelungstechnik. VDI Verlag, Düsseldorf.

Rill, G. (1994). Simulation von Kraftfahrzeugen. Vieweg, Braunschweig/Wiesbaden.

Soravia, P. (1996). $\mathcal{H}_{\infty}$ control of nonlinear systems: Differential games and viscosity solutions. SIAM J. Control and Optimization, 34(3), pp. 1071-1097.

Spencer Jr, B.F., Dyke, S.J., Sain, M.K. and Carlson, J.D. (1996). Modeling and control of magnetorheological dampers for seismic response reduction. Smart Materials and Structures, 5, pp. 565-575. Spencer Jr., B.F., Dyke, S.J., Sain, M.K. and Carlson, J.D. (1996). Phenomenological model of a magnetorheological damper. ASCE Journal of Eng. Mech. 123(3), pp. 1-9.

Stanway, R., Spronston, J.L and El-Wahed, A.K. (1995) Applications of electrorheological fluids in vibration control: a survey. Smart Material Structures 5, pp. 464-482.

Valásek, M., Novák, M., Sika, Z. and Vaculín, O. (1997). Extended ground-hook - new concept of semi-active control of truck's suspension. Vehicle System Dynamics, 27:5-6, pp. 289-303.

van der Schaft, A.J. (1991). $L_{2}$-gain analysis of nonlinear systems and nonlinear state feedback $H_{\infty}$ control. IEEE Trans. on Automatic Control, 37(6), pp. 770-784.

van der Schaft, A.J. (1996). $L_{2}$-Gain and Passivity Techniques in Nonlinear Control. Springer, Berlin.

Vögel, M., von Stryk, O., Bulirsch, R., Wolter, T.-M. and Chucholowski, C. (2003). An optimal control approach to real-time vehicle guidance. In: W. Jäger, H.-J. Krebs (eds.): Mathematics - Key Technologyfor the Future, Springer-Verlag, pp. 84-102.

von Stryk, O. (2001). User's Guide for DIRCOL Version 2.1: a direct collocation method for the numerical solution of optimal control problems. SIM, Technische Universität Darmstadt. World Wide Web: http://www.sim.informatik.tu-darmstadt.de/sw/dircol von Stryk, O. (2003). Numerical Hybrid Optimal Control and Related Topics. Habilitationsschrift, Technische Universität München.

Willems, J.C. (1972). Dissipative dynamical systems, part I: General theory. Arch. Rational Mech. Anal., 45, pp. 321-351. 